\section{Convergence Rates of Continuous-Time Stochastic ELS Parameter Estimation}

\section{HAN-FU CHEN AND JOHN B. MOORE}

tbstract-Discrete-time convergence rates for extended least squares (ELS) algorithms are generalized to the continuous-time case. An essential difference in the estimation is the appropriate prefiltering, while in the theory, the existence of solutions of the stochastic equations is a concern.

\section{INTRODUCTION}

Least squares (LS) estimation of continuous-time stochastic signal models with additive "white" noise is considered in [1]-[3]. Ergodicity assumptions are involved in the theory of [1]. In [2], [3] ergodicity is not assumed and convergence rates are given. In [1] there is a conjecture that corresponding convergence rates for extended least-squares algorithms in the colored noise case cannot be obtained. Some of the foundations are laid for such results in [2], [3]. In [4], ELS estimation is prescribed for signal models with appropriate prefiltering, including possibly the domination of certain noise signals by additive noise. Also, a weighting coefficient selection scheme is built into the ELS estimation to improve convergence properties and avoid finite escape times. The theory of [1][H] relies on Martingale convergence theorems.

Here, we generalize the work of [6] which gives rates of convergence for the discrete-time stochastic colored noise case to the continuous-time framework. A key ingredient is the prefiltering in the estimation, and for the theory, a key issue is the possibility of the existence of finite escape times on sample paths.

\section{Problem Statement}

Consider the dynamic system described by the following multivariable stochastic integral equation:

$$
A(S) y_{t}=B(S) u_{t}+C(S) v_{t}
$$

where $A(S), B(S)$, and $C(S)$ are matrix polynomials in the integral operator $S$ as

$$
\begin{aligned}
A(S)=I+A_{1} S+\cdots+A_{p} S^{p}, B(S) & =B_{1} S+B_{2} S^{2} \\
+ & \cdots+B_{q} S^{q}, C(S)=I+C_{1} S+\cdots+C_{r} S^{\prime} .
\end{aligned}
$$

Without loss of generality, $C(S)$ can be "minimum phase" in that $C(s)$ is full rank in $\operatorname{Re} s>0$ with $s$ the Laplace transform variable. For subsequent theory, we impose the stronger condition that

$$
\begin{gathered}
C(S) \text { is strictly minimum phase, in that } \\
C(s) \text { is full rank in } \operatorname{Re} s \geq 0 .
\end{gathered}
$$

Note that $S x_{t}=i_{0}^{t} x_{\lambda} d \lambda, S^{2} x_{t}=i_{0}^{i_{0}^{\tau}} x_{\lambda} d \lambda d \tau$. Here $v_{t}$ satisfies

$$
W(S) u_{1}=w_{1}
$$

where $\left(w_{f}, F_{l}\right)$ is a Wiener process, with $F_{l}$ a family of nondecreasing $\sigma$ algebras, and $W(S)=I+W_{1} S+\cdots+W_{r} S^{r}$ such that

$$
C(S) W^{-1}(S)-\frac{1}{2} I \text { is strictly positive real. }
$$

In [4], it is shown how such signal models can arise from plants, with

Manuscript received August 11. 1986.

H. -F. Chen is with the Institute of Systems Science, Academia Sinica, Beijing, China

J. B. Moore is with the Department of Systerns Engineering, Research School of Physical Sciences, Australian National University, Canberra. A.C.T., Australia. IEEE Log Number 8612539 the following transfer function description under zero initial conditions

$$
y^{p}(s)=\left[A^{p}(s)\right]^{-1} B^{p}(s) u^{p}(s)+\left[A^{p}(s)\right]^{-1} C^{p}(s) w^{p}(s)
$$

where $y_{t}^{p}$ are the outputs. $u_{r}^{p}$ the inputs, $w_{p}^{p}$ the noise disturbances, and $A^{p}(s) B^{p}(s), C^{p}(s)$ are polynomials in $s$. By filtering $y^{p}$, $u_{i}^{p}$ through asymptotically stable filters with transter function $\boldsymbol{W}^{\prime}\left(s^{-1}\right)$ to yicld $\boldsymbol{y}_{\text {, }}$, $u_{t}$ then the model for this system can be expressed in the form (2.1)-12.3). Also in [4], it is shown that by adding appropriate disturbances at the plant output, the model has the form $(2.1)-(2.3)$ with (2.4) satistied. Details are not repeated here.

Let us consider the estimation of the parameters $A_{i}, B_{i}, C_{i}$ on the busis of past measurements. $\left\{y_{r}, u_{r} ; \tau \leqq 1\right\}$, where

$$
\begin{aligned}
& u_{1} \text { is } F_{t}-\text { measurable and locally bounded in } L_{2} \text {. (That is } \\
& \int_{0}^{t}\left\|u_{\lambda}\right\|^{2} d \tau<\infty \quad \text { a.s. for all } t<\infty \text {.) }
\end{aligned}
$$

First, let us denote

$$
\begin{gathered}
\theta^{T}=\left[\begin{array}{lll}
-A_{1} \cdots-A_{p} & B_{1} \cdots & B_{q} C_{1} \cdots C_{r}
\end{array}\right] \\
\left(\phi_{1}^{0}\right)^{T}=\left[y_{l}^{T} S y_{l}^{T} \cdots S^{p-1} y_{l}^{T} \quad u_{?}^{T} \cdots S^{q-1} u_{l}^{T} v_{?}^{T} \cdots S^{r-1} v_{l}^{T}\right]
\end{gathered}
$$

so that the model $(2.1)$ can be written as

$$
y_{t}=S \theta^{T} \Phi_{1}^{0}+v_{t} .
$$

The ELS based estimation of $\theta$, yielding estimates $\theta_{1}$ involves the stochastic differential equation

$$
\begin{aligned}
& d \theta_{t}=\hat{P}_{t} \phi_{t} W(S)\left(d y_{t}-\theta_{1}^{T_{\phi}} \phi_{t} d t\right)^{T}, \quad \theta_{11} \text { arbitrary } \\
& \hat{P}_{f}=\left(\int_{0}^{t} 0, \phi_{f}^{\tau} d \tau+a^{-1} I\right)^{1} \quad a=\text { dimension of } \phi_{f} \quad(2,8)
\end{aligned}
$$

where

$$
\begin{gathered}
\phi_{i}^{T}=\left[y_{i}^{T} S y_{i}^{T} \cdots S^{p-1} y_{i}^{T} u_{i}^{T} \cdots S^{q-1} u_{i}^{T} \hat{i}_{i}^{T} \cdots S^{r-1} \hat{v}_{!}^{T}\right] \\
\hat{b}_{l}=y_{i}-S\left(\theta_{i}^{T} \phi_{i}\right) .
\end{gathered}
$$

Notice the presence of $W(S)$ and that $\operatorname{tr} \bar{P}_{1)}^{-1}=$

Assumption 1: The solution of (2.8) exists for almost all sample paths $\omega \in \Omega$ up to an escape time.

Let us define for each $\omega$

$$
\sigma=\sup \left\{t: \int_{0}^{t} 0 ; \bar{P}, 0, d \lambda<\infty\right\}
$$

When $\sigma$ is finite, it denotes a finite escape time of the process $\theta_{1}$. With the above definitions and assumption, we now claim that with $I_{|k<0|}$ the 2 dependent indicator function which is unity for $t<\sigma$ and zero otherwise. then

$$
\begin{gathered}
I_{|<<j|} \in F_{t} \\
\sigma \text { is a Markov time } \\
\int_{0}^{t}\left\|I_{\mid \lambda<0]} \bar{P}_{\lambda} \phi_{\backslash, \mid}\right\|=d \lambda<\infty \quad \text { for all finite } t .
\end{gathered}
$$

The result (2.12) follows since

$$
[t<\sigma] \doteq\left\{\omega: \int_{0}^{\prime} \phi_{\lambda}^{T} \bar{P}_{\lambda} \phi_{\lambda} d \lambda<\infty\right\} \in F_{1} .
$$

The result (2.13) follows since $[t<\sigma]=[\sigma \leqq t]^{c}$ where $c$ denotes the complement so that $[t \geqq \sigma] \in F_{1}$, or equivalently $\sigma$ is a Markov time [5]. The result $(2.14)$ follows from the property $(2.11)$, noting that $\bar{P}_{i} \leqq$ 
$\bar{P}_{\lambda}^{1 / 2} \bar{P}_{0} \bar{P}_{\lambda}^{1 / 2} \leqq\left(\right.$ tr $\left.\bar{P}_{0}\right) \bar{P}_{\lambda}$. It now proves convenient to consider the following modified form of (2.8):

$$
\begin{aligned}
& d \theta_{t}=I_{|t<\sigma|} P_{t} \phi_{r} W(S)\left(d y_{t}-\theta_{i}^{\Gamma} \phi_{i} d t\right)^{\Gamma}, \quad \theta_{0} \text { arbitrary } \\
& P_{t}=\left(\int_{0}^{t} I_{|<<|} \phi_{T} \phi_{,}^{T} d \tau+a^{-1} I\right)^{-1}, a=\text { dimension of } \phi_{t} .
\end{aligned}
$$

\section{Remarks:}

1) Should $\phi_{t}$ in $(2.8)^{\prime}$ be independent of $\theta_{t}$, then (2.11)-(2.14) hold and we could conclude that $\theta_{t}$ exists for all $t$ as the unique strong solution of

$$
\theta_{t}=\theta_{0}+\int_{0}^{t} I_{[\lambda<0]} P_{\lambda} \phi_{\lambda} W(S)\left(d y_{\lambda}-\theta_{\lambda}^{T} \phi_{\lambda} d \lambda\right)^{r}
$$

Since here for $C(S) \neq I, \phi$, is $\theta_{t}$ dependent, (2.8) is highly nonlinear, and we cannot conclude the same properties for $\theta_{t}$ without a formidable analysis of the nature of the nonlinearities. Assumption (2.10) in essence is that $\theta_{t}$ of $(2.8)^{\prime}$ exists for all $t$ as the unique strong solution of $(2.15)$.

2) In the case $C(S)=I$. the "white" noise case, $\phi_{t}=\phi_{t}^{0}$ is independent of $v_{t}, \hat{v}_{l}$ and hence of $\theta_{1}$. Moreover, under (2.5) from (2.10). $\sigma=\infty$ and there is no finite escape time aimost surely. Then $I_{[i<\sigma \mid} \equiv 1$ and (2.8) is a linear stochastic differential equation with a unique strong solution for all $t$.

3) For the case $W(S)=I$, the condition (2.4) can only be satisfied with $C(S)=I$ (in contrast to the discrete-time case). See also [2].

\section{Main Results}

Lemma 3.1: Consider the plant (signal model) (2.1)-(2.3) [or (2.6). (2.7)1, under conditions (2.4), (2.5). Consider also the ELS estimation scheme $(2.8)-(2.10)$. Then there are constants $\epsilon>0$ and $k_{1}>0$ such that

$$
\int_{0}^{1}\left[f_{\lambda}^{T} g_{\lambda}-\epsilon\left(g_{\lambda}^{T} g_{\lambda}+f_{\lambda}^{T} f_{\lambda}\right)\right] d \lambda+k_{\imath} \geqq 0 \quad \text { for } t<\sigma
$$

where, denoting $\theta_{t}=\theta-\theta_{t}, \tilde{v}_{t}=v_{t}-\hat{v}_{t}$

$$
g_{\imath}=\tilde{\theta}_{l}{ }_{\phi_{t}}, S f_{t}=[C(S)-W(S)] \tilde{u}_{t}+\frac{1}{2} S g_{l}
$$

Moreover, under condition (2.2), for some $k_{2}, k_{3}$, with $\bar{\phi}_{t}=\phi_{t}^{0}-\phi_{t}$

$$
\int_{0}^{1}\left\|\bar{\phi}_{\lambda}\right\|^{2} d \lambda \leqq k_{1} \int_{0}^{1}\left\|g_{\lambda}\right\|^{2} d \lambda+k_{3} \text {. }
$$

Proof: Simple manipulations yield for $t<\sigma$

$$
\begin{gathered}
d \tilde{u}_{t}=-\theta^{T} \tilde{\phi}_{t} d t-g_{t} d t, \text { or } C(S) \tilde{v}_{t}=-S g_{t} \\
d \tilde{\phi}_{t}=F \tilde{\phi}_{t} d t-G g_{\imath} d t
\end{gathered}
$$

where $\phi_{t}^{T}=\left[0 \cdots 0 \bar{v}_{t}^{T} \cdots S^{r-1} \bar{v}_{l}^{T}\right]$ and

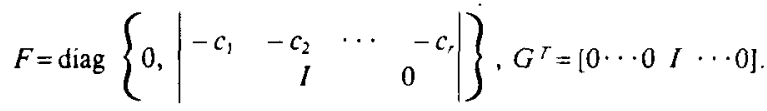

Also,

$$
\begin{aligned}
f_{t}=- & {[C(S)-W(S)] C^{-1}(S) g_{t}+\frac{1}{2} g_{t} } \\
= & {\left[W(S) C^{-1}(S)-\frac{1}{2} I\right] g_{t} . }
\end{aligned}
$$

Now (3.1) follows from (3.5) and application of the strict positive real condition (2.4). Also from (3.4) we see that $\phi_{l}$ are the states of a linear system with characteristic equation det $C\left(s^{-1}\right)=0$, so that result $(3.3)$ follows under (2.2).

Lemma 3.2: With the definition of $P_{t}$ in $(2.8)^{\prime}$ and $\sigma$ in (2.11),

$$
\int_{0}^{t} I_{|\lambda<0|} \phi_{\lambda}^{T} P_{\lambda} \phi_{\lambda} d \lambda=\ln \left(\operatorname{det} P_{i}^{-1}\right)+a \ln a
$$

Proof: From (2.8)

$$
\begin{aligned}
& \text { det } P_{, \cdot d t}^{\prime}=\operatorname{det} P_{1}^{-1} \operatorname{det}\left(I+I_{1, c, P} P, \phi, \phi^{\prime} d t\right) \\
& =\operatorname{det} P_{,}{ }^{1}\left(1+I_{\mid \cdots,+1} \phi_{;}^{\prime} P_{i} \phi_{i} d t\right)
\end{aligned}
$$

so that

$$
\frac{d\left(\operatorname{det} P_{t}^{\prime}\right)}{\operatorname{det} P_{t}^{-1}}=I_{!, \ldots, 1} \Phi, P_{;} O d t
$$

and the result (3.6) follows.

Lemma 3.3: Let the measurable process $M_{t}$ be adapted to $F_{l}$ and define

$$
T=\sup \left\{t: q_{l} \doteq \int_{0}^{1}\left\|M_{\lambda}\right\|^{2} d \lambda<\infty\right\} \text {. }
$$

Then for all $\eta>0$, as $t \rightarrow T$

$$
\int_{1}^{1} M_{\lambda} d w_{\lambda}=0\left[q_{1}^{1.2} \ln ^{1 / 2-\eta}\left(q_{l}+e\right)\right] \text { a.s. }
$$

Proof: As for $\sigma$ in (2.13), $T$ is a Markov time and $I_{1<T)} \in F_{t}$. Now for all $t$

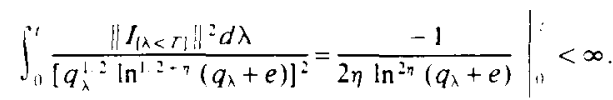

Let us define

$$
x_{i}=\int_{0}^{\prime} \frac{I_{[\lambda<T]^{3} M_{\lambda} d w_{\lambda}}}{q_{\lambda}^{1}{ }^{2} \ln ^{12 n}\left(q_{\lambda}+e\right)}
$$

and recall that under (3.8), via Martingale convergence. [4]

$$
\lim _{i \rightarrow \infty} x_{t}=x \text { a.s. }
$$

for some random variable $x$. Also, applying the Ito formula [5]

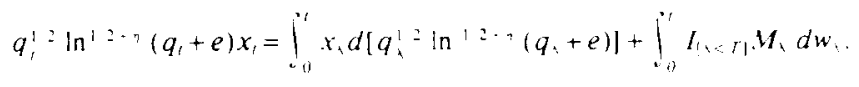

Now

$$
\begin{aligned}
& \frac{1}{q_{t}^{12} \ln 12-\eta\left(q_{t}+e\right)} \int_{0}^{1} I_{1 \nmid r \mid} M_{\downarrow} d w_{\lambda}
\end{aligned}
$$

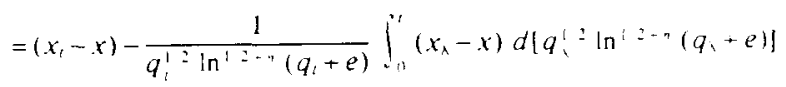

$$
\begin{aligned}
& \text { a.s. } \rightarrow\left\{\begin{array}{l}
o(1) \text { as } t \rightarrow \infty \text { if } q, \rightarrow \infty \\
0(1) \text { as } t \rightarrow \infty \text { otherwise. }
\end{array}\right.
\end{aligned}
$$

The result (3.7) follows.

Theorem 3.l: For the signal model/conditions (2.1)-(2.7) and ELS estimation scheme $(2.8)^{\prime}-(2.10)$, then as $t \rightarrow \sigma$

$$
\begin{aligned}
& \left\|\theta-\theta_{t}\right\|^{2}=0\left(\frac{\ln \lambda_{\max } P_{t}^{\prime}}{\lambda_{\min } P_{t}^{-1}}\right) \text { a.s } \\
& =0\left(\frac{\ln \lambda_{\max }\left(P^{\prime \prime}\right)^{-1}}{\lambda_{\min }\left(P^{\prime \prime}\right)^{1}-\ln \lambda_{\max }\left(P_{i}^{0}\right)^{-1}}\right) \text { a.s. (3.12b) }
\end{aligned}
$$

where $P_{l}^{0}$ is given from (2.8) ' with $\Phi_{l}$ replacing $\phi_{t}$. Moreover, on the set

$$
H_{1}=\left\{\omega: \limsup _{1 \rightarrow r} \frac{\ln \lambda_{\max } P_{i}^{-1}}{\lambda_{\min } P_{i}^{-1}}<\infty\right\}
$$




$$
H_{z}=\left\{\omega: \limsup _{i \rightarrow T} \frac{\ln \lambda_{\max }\left(P_{i}^{\prime}\right)^{-1}}{\lambda_{\operatorname{man}}\left(P_{i}^{(0)}\right)^{-1}-\ln \lambda_{\max }\left(P_{i}^{(0)}\right)^{-1}}<\infty\right\}
$$

then there is almost surely no finite escape time $(\sigma=\infty)$.

Proof: i) From (2.3). $W(s) d \hat{v}_{t}^{T}=W(S)\left(d v_{t}-d \tilde{v}_{t}\right]^{T}=\left[d w_{t}-\right.$ $W(S) d \tilde{u}^{T}$ l so that from $(2.8)^{\prime},(3.2),(3.4)$

$$
d \tilde{\theta}_{i}=-I_{\left\{1<\lambda_{1}\right.} P_{i} \phi_{i}\left\{\frac{1}{2} g_{t} d t+f_{t} d t+d w_{i}\right\}^{r} .
$$

Applying the Itô formula. then almost surely

$$
\begin{aligned}
& d\left(\operatorname{tr} \tilde{\theta}_{l}^{T} P_{t}^{-1} \tilde{\theta}_{l}\right)=-I_{|r<\sigma|}\left\{2 \operatorname{tr} \tilde{\theta}_{i}^{T} \phi_{i}\left(\frac{1}{2} g_{1} d t+f_{l} d t+d w_{l}\right)^{T}\right. \\
& \left.-\operatorname{tr} \tilde{\theta}_{i}^{T} \phi, \phi_{i}^{T} \tilde{\theta}_{t} d t-\phi_{l}^{T} P_{t} \phi, d t\right\} \\
& =-I_{l i<a]}\left\{2 f_{i}^{T} g_{t}+2 d w_{i}^{T} g_{t}-\phi_{i}^{T} P_{i} \phi_{t} d t\right\} \\
& \leqq-I_{\{<<\}}\left\{2 \epsilon\left(\left\|g_{l}\right\|^{2}+\left\|f_{l}\right\|^{2}\right) d t+2 g_{1}^{T} d w_{t}-\phi_{l}^{T} P_{t} \phi_{l} d t\right\}
\end{aligned}
$$

where the inequality follows from application of (3.1), under (2.4). Integrating and reorganizing gives, with $t \Lambda \sigma$ denoting $\min (t, \sigma)$

$$
\begin{aligned}
& 0 \leqq \operatorname{tr} \tilde{\theta}_{l}^{r} P_{l}^{-1} \tilde{\theta}_{t}+\epsilon \int_{0}^{r .1 \delta}\left(\left\|g_{\lambda}\right\|^{2}+\left\|f_{\lambda}\right\|^{2}\right) d \lambda \\
& \leqq \operatorname{tr} \tilde{\theta}_{0}^{T} P_{0}^{-1} \tilde{\theta}_{0}+2 k_{1}+\int_{0}^{i, a v} \phi_{\lambda}^{T} P_{\lambda} \phi_{\lambda} d \lambda \\
& -\left[\epsilon \int_{0}^{1.1 \sigma}\left\|g_{\lambda}\right\|^{2} d \lambda+2 \int_{0}^{+.1 v} g_{\lambda}^{r} d w_{\curlywedge}\right] \text {. }
\end{aligned}
$$

Now applying Lemma 3.3, as $t \rightarrow \sigma$, the last integral is dominated by the second last integral so that the square bracketed form becomes negative if $i_{1}^{\infty}\left\|g_{\lambda}\right\|^{2} d \lambda=\alpha$ and is of $O(1)$ if i is $\left\|g_{\lambda}\right\|^{2} d \lambda<\infty$. Noting this and applying Lemma 3.2 , as $t \rightarrow \sigma(3.15)$ leads to

$\int_{0}^{t h o}\left(\left\|g_{\lambda}\right\|^{2}+\left\|f_{\lambda}\right\|^{2}\right) d \lambda+\operatorname{tr} \tilde{\theta}_{t}^{r} P_{t}^{-1} \tilde{\theta}_{t}$

$$
=0(1)+\ln \left(\operatorname{det} P_{t}^{-1}\right) \text { a.s. }
$$

Now det $P_{i}^{-1} \geqq \lambda_{\max }\left(P_{l}^{-1}\right)(a)^{1-a} \geqq \operatorname{tr}\left(P_{l}^{-1}\right) a^{-a}$ so that

$$
\ln \operatorname{tr} P_{i}^{-1}-a \ln a \leqq \ln \operatorname{det} P_{i}^{-1} \leqq a \ln \operatorname{tr} P_{i}^{-1}
$$

Noting that $\|\tilde{\theta},\|^{2} \leqq\left[\lambda_{\min } P_{t}^{-1}\right]^{-1} \operatorname{tr} \theta_{t}^{T} P_{t}^{-1} \theta_{t}$, then (3.16), (3.17) lead to (3.12a)

ii) From (3.16), as $t \rightarrow \sigma$

$$
\int_{1}^{i}\left\|g_{\lambda}\right\|^{2} d \lambda=0\left(\ln \operatorname{tr} P_{i}^{-1}\right)
$$

Hence, by Lemma 3.1 , as $t \rightarrow \sigma$

$$
\int_{10}^{\prime}\left\|\bar{\theta}_{\lambda}\right\|^{2} d \lambda=0\left(\ln \operatorname{tr} P_{:}^{-1}\right)
$$

Thus, we have as $t \rightarrow \sigma$

$$
\begin{aligned}
\operatorname{tr} P_{t}^{-1} & \leqq 2 \operatorname{tr}\left(P_{l}^{0}\right)^{-1}+2 \int_{0}^{1}\left\|\tilde{\theta}_{\lambda}\right\|^{2} d \lambda \\
& =2 \operatorname{tr}\left(P_{t}^{0}\right)^{-1}+0\left(\ln \operatorname{tr} P_{t}^{-1}\right)
\end{aligned}
$$

which means that as $t \rightarrow \sigma$

$$
\left.\operatorname{tr} P_{i}^{-1}=0\left[\operatorname{tr}\left(P_{i}^{0}\right)^{-1}\right], \lambda_{\max } P_{i}^{-1}=0\left[\lambda_{\max } P_{i}^{0}\right)^{-1}\right]
$$

Further, for any $x \in R^{a}$ with $\|x\|=1$, we know that for $t<\sigma$

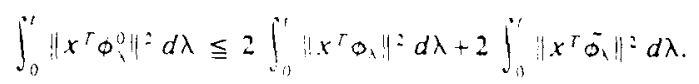

Selecting $x$ so that the first integral on the right-hand side is $\lambda_{\min }\left(P_{1}{ }^{1}\right)$. then the right-hand side is not less than $\lambda_{\min }\left(P_{1}^{0}\right)^{-1}$. Hence, for $t<\sigma$ and applying (3.18)

$$
\lambda_{\min }\left(P_{t}^{\prime \prime}\right)^{-1} \leqq 2\left\{\lambda_{\min }\left(P_{t}^{\prime}\right)+0\left[\ln \lambda_{\operatorname{mux}}\left(P_{t}\right)^{-i}\right\}\right.
$$

Thus, as $t \rightarrow \sigma$ and applying (3.19)

$$
\lambda_{\min }\left(P_{t}\right)^{-1} \geqq 0\left[\left(\lambda_{\min } P_{t}^{0-1}\right)-\ln \lambda_{\max }\left(P_{t}^{0}\right)^{-1}\right]
$$

Application of $(3.19),(3.20)$ in $(3.12 a)$ gives the result $(3.12 b)$.

iii) Now on the set $H_{i}$ of (3.13), from (3.12) $\left\|\theta_{t}\right\|$ does not diverge to $\infty$. Consequently, the system generating $\phi$, can be viewed as a linear timevarying system with parameters $\theta, \theta$, which do not diverge to $\infty$ on $H$. Thus, under (2.5) $\phi$, can grow no faster than exponentially and there is then no finite escape time on $H$.

Corollary 3.1: Consider the plant (signal model) (2.1) with $C(S)=1$, (2.3) with $W(s)=I$, so that $v_{\text {, }}$ is a Wiener process (and trivially (2.2), (2.4) are satisfied). Then $\phi_{f}=\phi^{0}$ and on the least squares estimation of $\theta$ via $(2.8),(2.9)$ under $(2.5)(2.10)$, there is almost surely no finite escape time, so $\sigma=\infty$ and $I_{\{<\sigma\}}=1$. Moreover. there is almost sure convergence of the parameter estimates $\theta_{t}$ given from (with $\bar{P}_{t}=P_{i}$ )

$$
\left\|\theta-\theta_{i}\right\|^{2}=0\left(\frac{\ln \lambda_{\max } P_{i}^{-1}}{\lambda_{\min } P_{i}^{-1}}\right) \text { as } t \rightarrow \infty .
$$

Proof: Since $\phi_{t}=\phi_{t}^{0}$ is derived from a linear time-invariant system with parameters $\theta, \phi^{0}$, can grow no faster than exponentially under $(2.5)$. and there is no finite escape time. The result $(3.21)$ now follows from an application of Theorem 3.1.

Remarks:

1) Under a plant stability assumption there is no finite escape time for $\phi^{0}$. and $\lambda_{\max }\left(P^{0}\right)^{-1} \leqq O(t)$. With $u_{t}$, $v_{\text {, suitably exciting so that }}$ $\lambda_{\min }\left(P_{r}^{0}\right)^{-1} \geqq 0(t)$. then $(3.18),(3.21)$ become

$$
\left\|\theta-\theta_{t}\right\|^{2}=0(t \text { in } t) \quad \text { as } t \rightarrow \infty .
$$

2) The result of Lemma 3.3 is perhaps of independent interest to.any ELS stochastic analysis.

\section{CONCLLSION}

Convergence (and divergence) rates for ELS estimation of a class of linear continuous-time stochastic signal models have been demonstrated. Assuming the nonlinear stochastic differential equations involved have solutions which exist up until (sample path dependent) finite escape times. conditions are derived which exclude the existence of tinite escape times.

The material of this note complements derivations of a companion paper which employ weighting coefficient selections to avoid tinite escape times. and gives global convergence rates for continuous-time ELS based stochastic adaptive control [4].

\section{REFERENCES}

(1] J. H. Van Schuppen, "Convergence results for continuous time adaptive stochastk tiltering algorithms." J. Math. Anal. Appl., vol. 96. no. 1. pp. 209-225. 1983

(2) H. F. Chen, Recursive Estimation and Control for Stochastic Systems. Vien York: Wiley. 1985.

[3] _- "Quasi-least-squares identification and its strong consistency," Int. J. Contr., vol. 34, no. 5, pp. 921-936. 1981 .

[4] J. B. Moore, "Convergence of continuous time stochastic adaptive ELS estimation and control schentes," submitted for publication.

[5] R. S. Liptser and A. N. Shiryayev. Statistics of Random Processes: I. General Theory. New York: Springer-Verlag. 1977.

[6] H. F. Chen and L. Guo. "Convergence rate of least squares identification and adaptive control for stochastic systems." Int. J. Contr., pp. 1459-1476. Yov 1986. 